citable using Digital Object Identifier - DOI)

Phys. Status Solidi A, 1-7 (2016) / DOI 10.1002/pssa.201600134

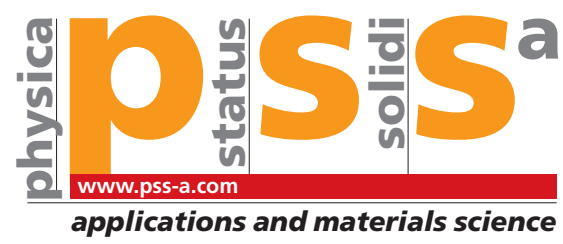

\title{
Mid-infrared thin-film diamond waveguides combined with tunable quantum cascade lasers for analyzing the secondary structure of proteins
} \author{
Fredrik Nikolajeff $^{2,3}$, Lars Österlund ${ }^{2,3}$, and Boris Mizaikoff ${ }^{1}$ \\ ${ }^{1}$ Institute of Analytical and Bioanalytical Chemistry, Ulm University, 89081 Ulm, Germany \\ ${ }^{2}$ Department of Engineering Sciences, Uppsala University, Box 534, 75121 Uppsala, Sweden \\ ${ }^{3}$ Molecular Fingerprint Sweden AB, Eksätravägen 130, 75655 Uppsala, Sweden
}

Ángela I. López-Lorente ${ }^{*, 1}$, Pei Wang ${ }^{1}$, Markus Sieger', Ernesto Vargas Catalan ${ }^{2}$, Mikael Karlsson $^{2,3}$,

Received 4 March 2016, revised 2 April 2016, accepted 4 April 2016

Published online 4 May 2016

Keywords diamond, infrared sensors, infrared spectroscopy, proteins, quantum cascade lasers, waveguides

*Corresponding author: e-mail: angela.lopez-lorente@uni-ulm.de, Phone: +49 73150 22750, Fax: +49 7315022763

Diamond has excellent optical properties including broadband transmissivity, low self-absorption, and a high refractive index, which have prompted its use for optical sensing applications. Thinfilm diamond strip waveguides (DSWGs) combined with tunable quantum cascade lasers (tQCLs) providing an emission wavelength range of 5.78-6.35 $\mu \mathrm{m}\left(1735-1570 \mathrm{~cm}^{-1}\right)$ have been used to obtain mid-infrared (MIR) spectra of proteins, thereby enabling the analysis of their secondary structure via the amide I band. Three different proteins were analyzed, namely bovine serum albumin (BSA), myoglobin, and $\gamma$-globulin. The secondary structure of BSA and myoglobin has a major contribution of $\alpha$-helices, whereas $\gamma$-globulins are rich in $\beta$-sheet structures, which is reflected in the amide I band. A comparison of the spectra obtained via the combination of the TQCL and DSWG with spectra obtained using conventional Fourier transform infrared (FTIR) spectroscopy and a commercial diamond attenuated total reflection (ATR) element has been performed. It is shown that the main features evident in FTIR-ATR spectra are also obtained using tQCL-DSWG sensors.

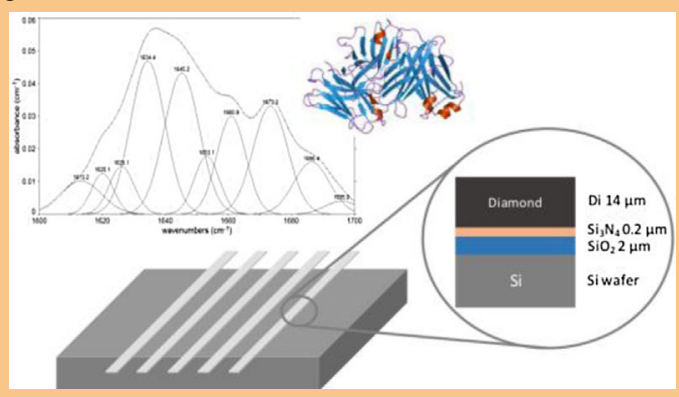

1 Introduction In mid-infrared (MIR) spectroscopy and sensing, vibrational and rotational modes of molecules of both organic and inorganic nature are excited revealing structural information with inherent molecular selectivity [1]. Specifically, infrared spectroscopy can provide information on protein structures, and has been widely used for protein analysis via the vibrational resonances arising from their polypeptide backbone or side chains $[2,3]$. Among the infrared spectroscopic techniques, attenuated total reflection (ATR) spectroscopy has emerged as a most useful technique for protein analysis.
Attenuated total reflection fiber optics have been used as MIR transducers, especially for analyzing liquid samples via the evanescent field generated by internal total reflection [4]. The evanescent electromagnetic field couples with IR absorbing analytes (i.e., proteins) at the surface of the waveguide, and the field decays exponentially a couple of micrometers into the adjacent medium/sample. Such interaction leads to an IR-ATR absorption spectrum characteristic of the protein. A major limitation of conventional MIR spectroscopy regarding protein analysis is the sensitivity. Recently, emerging light sources such as quantum cascade 
lasers (QCLs) are promising miniaturized IR sensing schemes [5] particularly useful for biomolecular analysis due to their high optical output power, narrow spectral bandwidth, small dimensions, long lifetime, and broadly tunable emissions (i.e., coupled to an external cavity and grating up to $300 \mathrm{~cm}^{-1}$ ) [6]. Although the use of externalcavity QCLs for the analysis of proteins in aqueous solution has been described, only MIR transmission measurements have been reported to date with the QCL enabling an extended absorption path length [7]. In this context, continuous wave (cw) operation of quantum cascade lasers shows substantial potential for chemical sensing applications [8].

Infrared transmitting waveguides (i.e., predominantly fiberoptic structures) are usually fabricated from IRtransparent materials [9] including polycrystalline silver halides [10-12], amorphous chalcogenide glasses [13, 14], heavy metal fluorides [15], tellurium halides [16, 17], single-crystalline sapphire [18, 19], and hollow waveguide structures [20-22]. Recently, semiconductor waveguides enabling on-chip MIR sensing schemes have been described including different optical configurations such as planar $\mathrm{GaAs} / \mathrm{Al}_{0.2} \mathrm{Ga}_{0.8} \mathrm{As}$ slab waveguides [23], GaAs/ $\mathrm{Al}_{0.2} \mathrm{Ga}_{0.8} \mathrm{As}$ strip/ridge waveguides [24], and $\mathrm{HgCdTe}$ waveguide structures [25].

Also in the MIR, diamond has excellent optical properties such as low self-absorption and scattering, and transparency over a wide spectral range, which - combined with its high refractive index and its hardness and chemical resistance render it an ideal material for chem/bio sensing applications in the MIR regime. The combination of tunable quantum cascade lasers (tQCLs) with thin-film diamond strip waveguides (DSWGs) was recently demonstrated for chemical sensing, thereby revealing a significant sensitivity improvement compared to conventional MIR waveguides [26].

In the present study, the suitability of thin-film diamond strip waveguides for analyzing protein spectra was investigated. While further studies are needed in order to increase the sensitivity of the measurements (i.e., by attaching appropriate biological or biomimetic recognition elements at the surface of the DSWG or by optimizing the dimensions of the waveguide), these preliminary results clearly reveal the potential of the combination of DSWGs with tQCLs. A tQCL was operated in continuous wave mode, and tuned within a spectral window of 1735$1570 \mathrm{~cm}^{-1}$ for analyzing the secondary structure of proteins using only a minute volume of sample.

\section{Experimental}

2.1 Protein preparation Three different proteins were analyzed in this study: albumin from bovine serum (BSA) (Sigma A9418), $\gamma$-globulin (Sigma G7516), and myoglobin (Sigma M0630), all of them provided by SigmaAldrich Co. (St. Louis, MO). Stock solutions of each protein at a $40 \mathrm{mg} \mathrm{mL}^{-1}$ concentration were prepared in ultrapure water. For infrared analysis, diluted solutions of the proteins were prepared in phosphate buffer saline (PBS) media ( $\mathrm{pH} 7.0)$.
2.2 Fabrication of the diamond waveguides Diamond waveguides were fabricated by thin-film deposition with subsequent optical photolithography and inductively coupled plasma etching (ICP) to obtain laterally defined thin-film diamond waveguides, as previously described [26]. Firstly, microwave plasma-assisted chemical vapor deposition (CVD) was used to grow a 14- $\mu \mathrm{m}$ thick diamond film on top of a $200-\mathrm{nm} \mathrm{Si}_{3} \mathrm{~N}_{4}$ layer and a $2-\mu \mathrm{m}$ $\mathrm{SiO}_{2}$ cladding layer deposited onto a $\mathrm{Si}$ wafer substrate (from Diamond Materials $\mathrm{GmbH}$ ) with a thickness of $1 \mathrm{~mm}$. $\mathrm{SiO}_{2}(n \approx 1.46)$ and $\mathrm{Si}_{3} \mathrm{~N}_{4}(n \approx 2)$ were added as cladding layer in order to confine wave propagation within the diamond waveguide due to the mismatch in refractive index of diamond $(n \approx 2.38)$ and $\mathrm{Si}(n \approx 3.45)$ in the wavelength range of interest. In addition, $\mathrm{Si}_{3} \mathrm{~N}_{4}$ also promotes the adhesion of the diamond film. The Si wafer was thinned down to $600 \mu \mathrm{m}$ after the diamond deposition and subsequent polishing, and then, cut into pieces of $1 \mathrm{~cm}^{2}$ for fabricating individual thin-film diamond waveguide strips. All samples were cleaned in a $\mathrm{H}_{2} \mathrm{O}_{2}$ and $\mathrm{H}_{2} \mathrm{SO}_{4}$ solution, and subsequently rinsed with isopropanol.

The $1 \mathrm{~cm}^{2}$ segments were coated with Al. A $4 \mu \mathrm{m}$ thick Al film was sputtered on the diamond, after which the waveguide geometry was defined in Shipley S1813 photoresist by a standard UV-lithography process. The Al film was etched in $\mathrm{BCl}_{3} / \mathrm{Cl}_{2}$ plasma to make a mask for the subsequent diamond etching. The diamond was etched in $\mathrm{Ar} / \mathrm{O}_{2} / \mathrm{SF}_{6}$ plasma, which resulted in inclined sidewalls as seen in Fig. 3c. The sample was subsequently turned over, and an Al mask was defined in a similar manner on the backside. The silicon substrate was then etched in a standard Bosch process, leaving only a narrow $\mathrm{Si}$ frame for the waveguides to rest on. Leftover mask material was removed in piranha solution. A SEM image of the resulting freehanging DSWG is shown in Fig. $3 \mathrm{~d}$.

2.3 Experimental setup The experimental set up comprised a broadly tunable QCL (tQCL) (Daylight Solutions, Inc., San Diego, CA) used as MIR light source providing radiation in the spectral window of 5.78-6.35 $\mu \mathrm{m}$ (1735-1570 $\mathrm{cm}^{-1}$ ) (Fig. 1). The laser radiation was focused

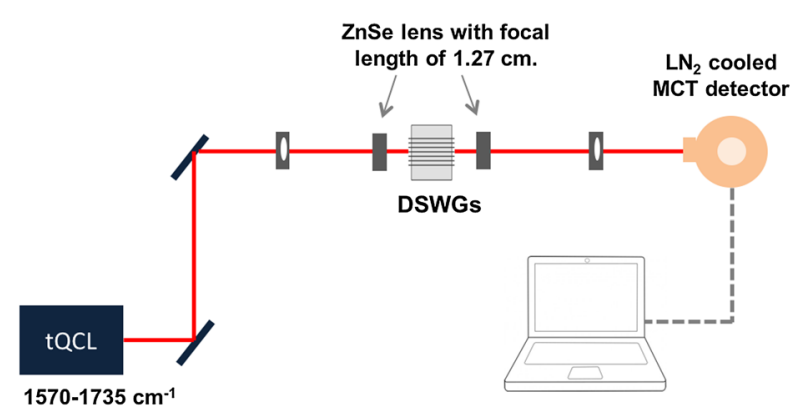

Figure 1 Scheme of the experimental set up for MIR measurements comprising a tunable quantum cascade laser (tQCL), mirrors, $\mathrm{ZnSe}$ lenses, the chip with the diamond strip waveguides, and an MCT detector. 
onto the in-coupling facet of the diamond waveguides via a ZnSe lens with a focal length of $1.27 \mathrm{~cm}$. The DSWG was located on a 3D translational stage enabling alignment of the QCL radiation to the in-coupling facet of the waveguide. MIR light propagated through the diamond core layer to the end facet of the waveguide. A ZnSe lense doublet focused the emitted light at the distal end of the DSWG onto a liquid-nitrogen-cooled mercury-cadmium-telluride (MCT) detector element (Kolmar Technologies, Newbury, MA) connected to an oscilloscope. The signal was recorded using a LabView program for further data processing.

The tQCL was operated in continuous wave $(\mathrm{cw})$ mode with a current of $650 \mathrm{~mA}$, and scanning across the spectral range of $1720-1590 \mathrm{~cm}^{-1}$ at a resolution of $1 \mathrm{~cm}^{-1}$. The QCL was cooled at approximately $20{ }^{\circ} \mathrm{C}$ during operation. All experiments were performed at ambient conditions. A volume of $5 \mu \mathrm{L}$ of protein solution was added at the center of the DSWG surface. The surface tension spread the droplet across the waveguide surface covering the entire beam waist. Proteins interacted with the evanescent field attenuating the radiation at the respective vibrational resonances, and gave rise to an IR-ATR absorption spectrum. Protein solutions were removed from the DSWG using lens-cleaning tissue, and the waveguide surface was subsequently cleaned with $10 \mathrm{mmol} \mathrm{L}^{-1}$ sodium dodecyl sulfate (SDS), distilled water, and acetone.

2.4 Conventional FTIR-ATR system In order to compare the results obtained with the DSWG, the infrared spectra of protein samples were also recorded using a Bruker Vertex 70 FTIR spectrometer (Bruker Optik, GmbH, Ettlingen, Germany) equipped with an attenuated total reflection unit (ConcentratIR2; Harrick Scientific Products, New York) comprising a diamond ATR (10 reflections) sampling plate connected to $\mathrm{ZnSe}$ radiation coupling optics. The signal is recorded at a liquid nitrogen cooled MCT detector.

Measurements were carried out placing $20 \mu \mathrm{L}$ of the sample on the ATR cell unit. Each spectrum was an average of 1000 sample scans with a spectral resolution of $2 \mathrm{~cm}^{-1}$ within the spectral range of $4000-800 \mathrm{~cm}^{-1}$. Background spectra of PBS buffer were acquired and subtracted from the protein sample spectra. Average protein spectra of at least three independent measurements were calculated in order to ensure reproducibility. After each measurement, the ATR cell was cleaned with $10 \mathrm{mmol} \mathrm{L}^{-1} \mathrm{SDS}$ solution followed

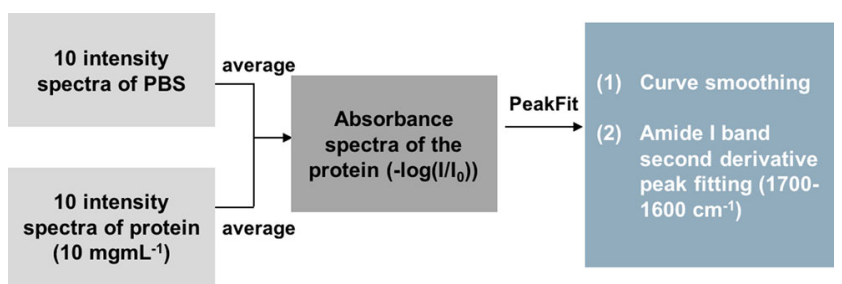

Figure 2 Scheme of the data treatment performed for the analysis of the amide I band of proteins using the combination of DSWGs and tQCL. by distilled water. Data acquisition was performed using the OPUS 6.5 software package (Bruker Optik).

2.5 Data analysis In order to ensure reproducibility of the measurements carried out with the DSWG combined with the QCL setup 10 spectra each of PBS buffer - used as reference - and of the protein sample solutions were acquired and the average was calculated. The intensity of the radiation propagating through the waveguide was dampened via evanescent field absorption following $A=-\log \left(I / I_{0}\right)$, where $I$ was the average of 10 measurements of the protein sample, and $I_{0}$ the average of 10 spectra of PBS buffer. Then, spectra were smoothed with $20 \%$ fast fourier transform (FFT) filtering using PeakFit software (Systat Software Inc., San Jose, CA). FFT filtering removes any linear trend, which might appear as a low frequency component; thus, the frequency domain noise is reduced for the dataset. The percentage of smoothing defines the breadth of the smoothing or filtering window. For FFT filtering, the percentage of smoothing controls the channels that are zeroed in the frequency domain. The amide I band (1700$1600 \mathrm{~cm}^{-1}$ ) of each protein spectrum was analyzed using second derivative and Gaussian peak fitting performed via PeakFit software (Fig. 2).

Protein spectra analyzed with the conventional FTIR spectrometer were subject to a similar treatment routine, as previously described [27]. IR spectra were acquired and processed with Opus 6.5 software, which was also used to exclude water vapor and $\mathrm{CO}_{2}$ interferences. The spectra were then smoothed using a 13-point Savitzky-Golay algorithm. The baseline of each spectrum was corrected in the wave number region of 4000-3950 and 2450$2400 \mathrm{~cm}^{-1}$ using PeakFit software. Finally, the amide I region of the spectrum resulting from the average of three independent measurements was fitted using second derivatives similar to the procedure used for DSWG measurements after $20 \%$ FFT smoothing.

\section{Results and discussion}

3.1 Characterization of the DSWGs Figure 3 shows optical and scanning electron microscopy (SEM) images of the fabricated diamond waveguides. As previously described, they comprise a $14 \mu \mathrm{m}$ diamond film

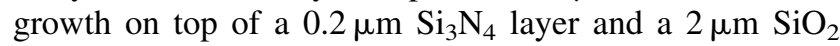
cladding layer on a $\mathrm{Si}$ wafer substrate. The Si wafer contains strips of different width, i.e., 100, 250, and $500 \mu \mathrm{m}$. For the measurements herein, strips with a width of $500 \mu \mathrm{m}$ were employed. The length of the waveguide is approximately $8 \mathrm{~mm}$. The waveguides support multimode propagation (i.e., up to six TE and TM modes) at the wavelength range of interest (i.e., $1720-1590 \mathrm{~cm}^{-1}$ ).

3.2 Optimization of the measurement conditions The variables that may influence the measurements using the combination of the diamond waveguides with the QCL were investigated. Spectra were recorded at different spectral resolutions in the range of $0.2-2 \mathrm{~cm}^{-1}$ 

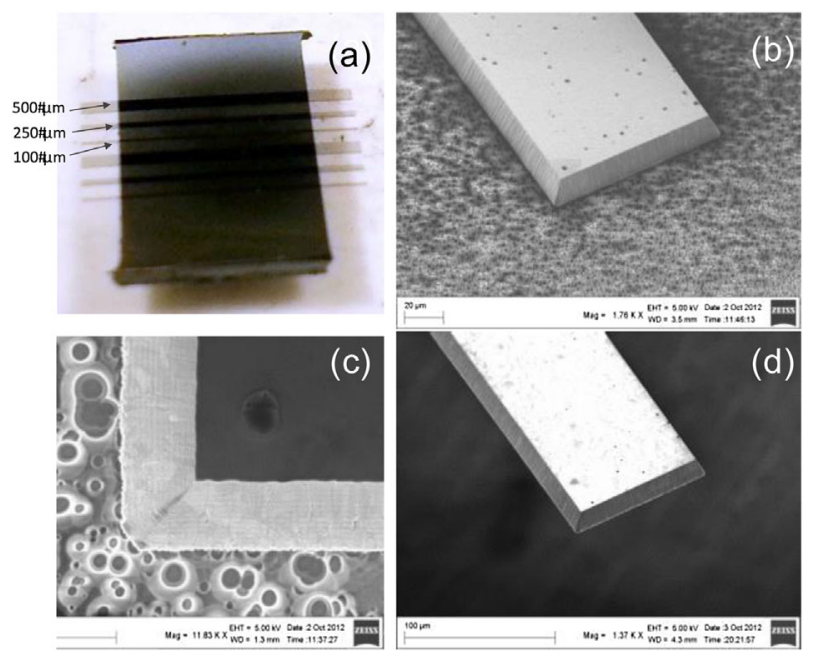

Figure 3 Diamond strip waveguides (DSWGs) grown on a silicon wafer substrate: (a) optical image of the diamond waveguides with different strip widths $(100,250$, and $500 \mu \mathrm{m})$, (b) SEM close-up of the in/out-coupling end facet of a DSWG with a thickness of $14 \mu \mathrm{m}$ on the Si wafer, (c) top-view SEM image of the beveled DSWG edge structures, and (d). SEM image of the free-hanging DSWG after Si substrate removal.

averaging between 1 and 10 spectra. The finally selected conditions in terms of sensitivity and measurement time were $1 \mathrm{~cm}^{-1}$ spectral resolution averaging 10 spectra. The quantum cascade laser may operate in both pulse and continuous wave mode. During initial studies, cw operation at a current of $650 \mathrm{~mA}$ was selected. Spectra of proteins were studied in water, deuterium oxide, and PBS buffer media. Finally, PBS buffer ( $\mathrm{pH} 7$ ) was used with the neat PBS buffer solution serving as reference.

3.3 Analysis of the secondary structure of proteins The secondary structures of three different proteins - bovine serum albumin, myoglobin, and $\gamma$ globulin - were investigated. Proteins were diluted in PBS to a concentration of $10 \mathrm{mg} \mathrm{mL}^{-1}$, and their spectra were recorded via DSWG coupled to the tQCL in the range 1720$1590 \mathrm{~cm}^{-1}$. Within the IR spectrum of proteins, the amide I band $\left(1700-1600 \mathrm{~cm}^{-1}\right)$ provides information on the secondary structure of proteins in a label-free fashion.

Figure 4 depicts the curve fitting of the amide I band for each of the averaged protein spectra $(n=10)$. The spectra were fitted by Gaussian peaks. Evidently, the amide I region BSA is characterized by peaks approximately at $1651 \mathrm{~cm}^{-1}$ owing to the predominant presence of $\alpha$-helix structures. Myoglobin also shows abundant $\alpha$-helix structures via bands around $1655 \mathrm{~cm}^{-1}$. On the other hand, $\gamma$-globulin is rich in $\beta$-sheet structures, and the amide I band is correspondingly more pronounced at approximately $1639 \mathrm{~cm}^{-1}$. These results are in agreement with previous studies carried out on silicon ATR waveguides [27].

3.4 Comparison of measurements with a conventional diamond ATR crystal A comparison with
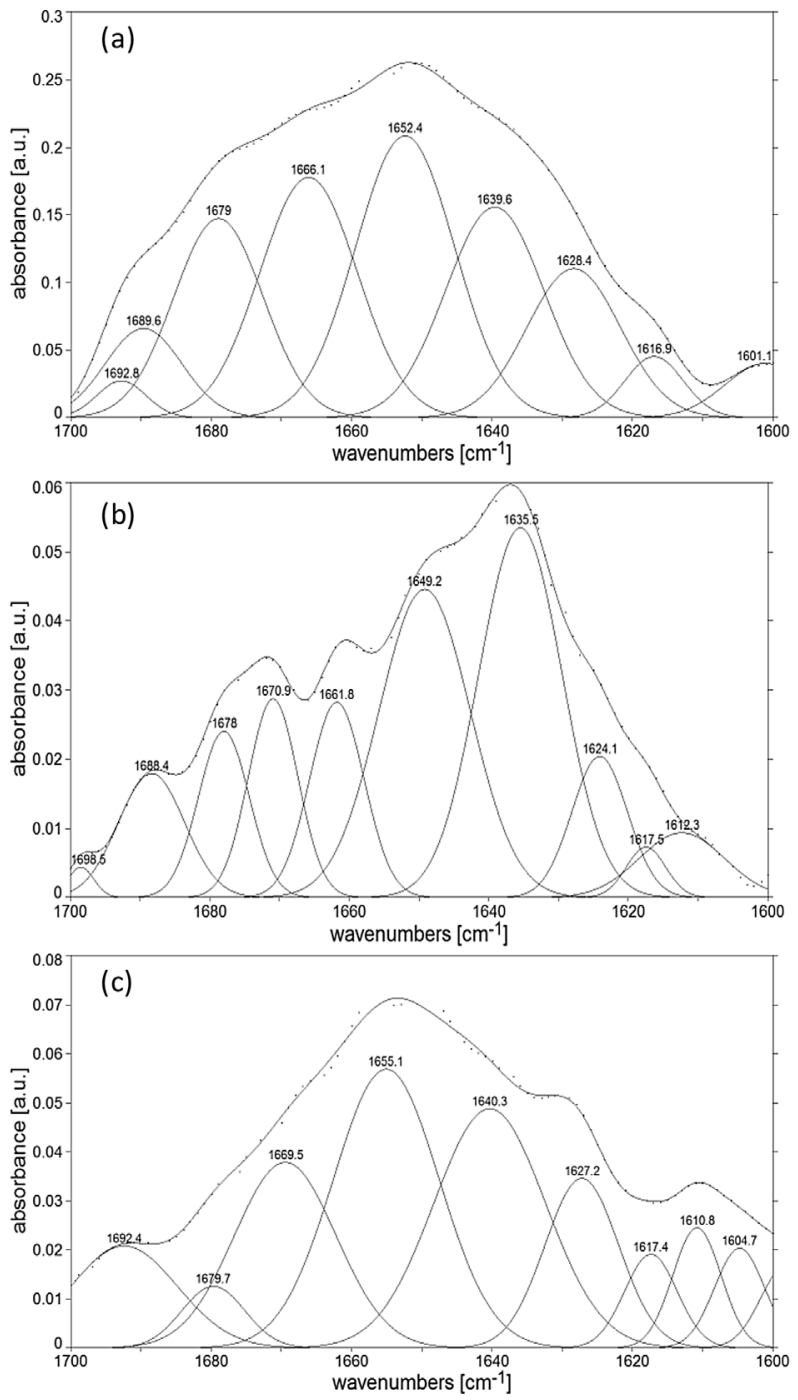

Figure 4 Curve fitting of protein amide I band of averaged ( $n=10) 10 \mathrm{mg} \mathrm{mL}^{-1}$ protein spectra in PBS (a) bovine serum albumin, (b) $\gamma$-globulin, and (c) myoglobin, respectively. The spectra were fitted by Gaussian peak fitting.

spectral features obtained with a conventional diamond IR-ATR crystal using a FTIR spectrometer equipped with an attenuated total reflection unit was carried out. Figure 5 shows the amide I band curve fitting for BSA and $\gamma$ globulins proteins obtained with the two different devices. For simplification, the spectra of myoglobin protein are not shown, but a similar correlation between the spectra obtained using the QCL-DSWG and the FTIR diamond and silicon waveguides was observed. The absorbance achieved with the QCL-DSWG combination is higher than the one observed in conventional IR-ATR spectroscopy using a diamond waveguide. However, it has to be considered that the concentration used for the QCL-DSWG measurements is $20 \times$ higher. Nevertheless, an around 4-fold sensitivity gain is observed with the QCL-DSWG setup compared to the FTIR analysis. On the other hand, as 

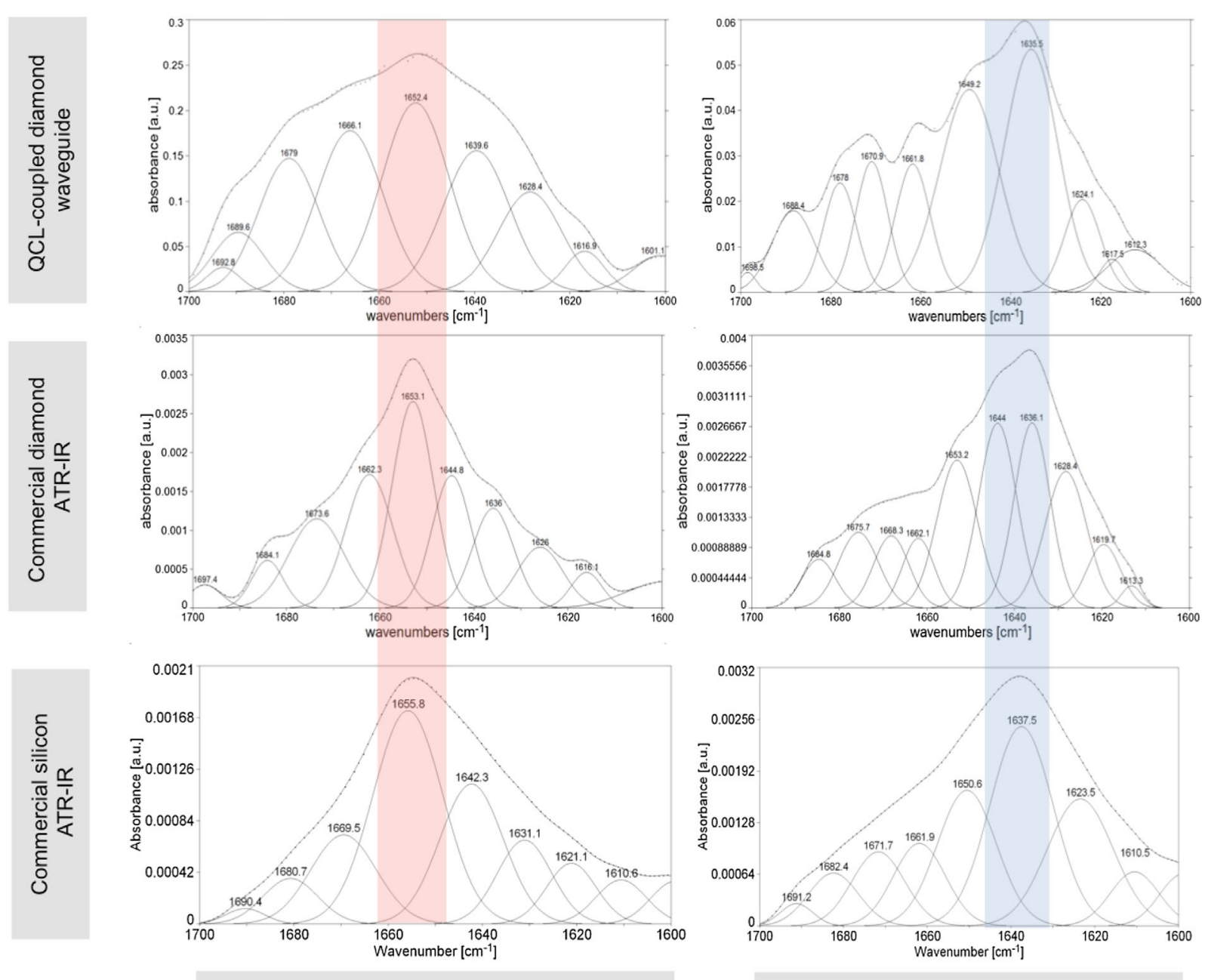

Bovine serum albumin

$\gamma$-globulins

Figure 5 Curve fitting of protein amide I band of BSA and $\gamma$-globulin proteins acquired with the tQCL coupled with the DSWG (curve fitting of averaged $(n=10) 10 \mathrm{mg} \mathrm{mL}^{-1}$ protein solution in PBS), a commercial diamond ATR crystal (curve fitting of averaged $(n=3)$ $0.5 \mathrm{mg} \mathrm{mL}^{-1}$ protein solution in PBS) and a commercial silicon ATR crystal (curve fitting of averaged $(n=4) 0.5 \mathrm{mg} \mathrm{mL} \mathrm{mrotein}^{-1}$ solution in PBS).

can be seen in Fig. 5, after curve fitting both spectra show a major contribution of $\alpha$-helix and $\beta$-sheet to the amide I band of BSA and $\gamma$-globulin, respectively. However, some differences in peak shape among them were observed. As shown in Fig. 5, in the case of BSA the spectrum obtained with the QCL combined with the DSWG is broader compared to those recorded via FTIR, although the main feature is observed at similar wavenumbers as a consequence of the major contribution of $\alpha$-helix structures to the secondary structure of the protein. Regarding $\gamma$ globulin, in both spectra obtained with the FTIR spectrometer and the QCL-DSWG combination, the amide I band shows the more prominent feature at around $1640 \mathrm{~cm}^{-1}$. The main features observed in the FTIR spectra are also observed in the spectra obtained via the QCLDSWG system. As observed herein, slight differences in the shape of the featured bands have also been documented in literature [26, 28, 29], i.e., ascribed to residual $\mathrm{H}_{2} \mathrm{O}$ absorption affecting the QCL measurements [28].

Considering the difference in conditions at which both spectra were recorded, such minute differences in the shape of the amide I band are expected. In addition, the physical nature of the light sources and how they provide wavelength-dispersed spectra are fundamentally different, which leads to different signal generation mechanisms. While FTIR spectrometers obtain information from all wavelengths simultaneously due to the modulation via the interferometer and subsequent Fourier transformation of the time-domain signal into the frequency domain, continuous wave operated QCLs continuously tune through their emission window in a consecutively fashion.

Furthermore, the ATR waveguides are different. Although in the case of the conventional ATR diamond crystal used in the FTIR 10 macroscopic reflections occur, 
in the case of the DSWGs multimode propagation (up to six TE and TM modes) in a thin-film conduit is the waveguiding mechanism, which may also affect the recorded spectrum due to beat patterns and potentially varying power per wavelength. Finally, it should be also noted the experimental setup for the QCL-DSWG measurement is entirely exposed to the open atmosphere, thus, influences of humidity and temperature may be more pronounced.

In addition, IR-ATR spectra of proteins may be affected by adsorption kinetics [30]. The different duration of measurements with the different experimental setups may also contribute to some structural changes observed within the spectra. However, since the duration of the measurements was quite similar during all experiments, and since adsorption of proteins occurs within seconds [31], such contributions are expected to be minute.

Last but not least, the volume and concentration of proteins should be also considered. For FTIR measurements, $20 \mu \mathrm{L}$ of a $0.5 \mathrm{mg} \mathrm{mL}^{-1}$ sample were used, whereas with the QCL-DSWG combination $5 \mu \mathrm{L}$ of sample were sufficient at a concentration of $10 \mathrm{mg} \mathrm{mL}^{-1}$. The use of different concentrations and volume of proteins may also lead to different protein arrangements at the waveguide surface. The hypotheses on the potential origin of the differences observed in both kinds of spectra, FTIR and tQCL, will subject of future investigations.

The influence of the waveguide material was further investigated by recording FTIR spectra of BSA and $\gamma$ globulin using a diamond and a silicon waveguide, which support 10 and 11 reflections, respectively. These measurements were carried out with the same equipment (i.e., ATR system and FTIR), thus any influence of light source, detector, mirrors, etc. was avoided with the only difference being the material of the waveguide. For the same concentrations and sample volumes, Fig. 5 shows differences in the shape of the amide I band, although the main features, which depend on the secondary structure of the proteins appear at similar wavenumbers. Diamond and silicon have different refractive indices (i.e., $n \cong 2.4$ and 3.45, respectively), hence, the evanescent field generated within the diamond ATR waveguide has a higher penetration depth than that emanating at the silicon ATR surface. As anticipated, an increase in the sensitivity of the FTIR measurements of the studied proteins was observed using the diamond ATR waveguide.

4 Conclusions The potential of thin-film diamond strip waveguides coupled to tunable quantum cascade lasers for the MIR spectroscopic study of the secondary structure of proteins has been investigated. The spectra in the amide I region (1700-1600 $\mathrm{cm}^{-1}$ ) of three different proteins were recorded in PBS media. The contribution of the different secondary structures, i.e., $\alpha$-helix and $\beta$-sheets was readily observed. Advantageously, MIR measurements using DSWGs were performed with minute amounts of sample (i.e., $5 \mu \mathrm{L}$ ).
Spectra obtained with the tQCL-DSWG combination were compared with FTIR-based spectra using a conventional diamond ATR waveguide. The main features observed in the FTIR spectra were reproduced within the DSWG spectra. The observed differences in peak shape were attributed to the different light sources, and nature of the waveguides. Nevertheless, the combination of DSWGs with state-of-the-art light sources such as tunable QCLs operating in the spectral region of interest is a promising IR chem/bio sensing technology that may flexibly be adapted to a wide range of MIR sensing scenarios addressing relevant biomolecular constituents such as proteins.

Acknowledgments Authors wish to thank the Horizon 2020 Framework Programme of the European Union for funding within the MSCA RISE Project TROPSENSE. A.I. López-Lorente also wishes to thank the Alexander von Humboldt Foundation for the award of a Postdoctoral Fellowship at the Institute of Analytical and Bioanalytical Chemistry (Ulm University, Germany).

\section{References}

[1] X. Wang, M. Sieger, and B. Mizaikoff, Proc. SPIE 8631, 86312M (2013).

[2] A. I. López-Lorente and B. Mizaikoff, Anal. Bioanal. Chem. 408, 2875-2889 (2016).

[3] R. Lu, W. Li, A. Katzir, Y. Raichlin, H. Yu, and B. Mizaikoff, Analyst 140, 765-770 (2015).

[4] R. Lu, G. Sheng, W. Li, H. Yu, Y. Raichlin, A. Katzir, and B. Mizaikoff, Angew. Chem. Int. Ed 52, 2265-2268 (2013).

[5] J. Faist, F. Capasso, D. L. Sivco, C. Sirtori, A. L. Hutchinson, and A. Y. Cho, Science 264, 553-556 (1994).

[6] C. Young, S. S. Kim, Y. Luzinova, M. Weida, D. Arnone, E. Takeuchi, T. Day, and B. Mizaikoff, Sens. Actuators B 140, 24-28 (2009).

[7] M. R. Alcaráz, A. Schwaighofer, C. Kristament, G. Ramer, M. Brandstetter, H. Goicoechea, and B. Lendl, Anal. Chem. 87, 6980-6987 (2015).

[8] M. Beck, D. Hofstetter, T. Aellen, J. Faist, U. Oesterle, M. Ilegems, E. Gini, and H. Melchior, Science 295, 301-305 (2002).

[9] B. Mizaikoff, Anal. Chem. 75, 258A-267A (2003).

[10] M. Karlowatz, M. Kraft, and B. Mizaikoff, Anal. Chem. 76, 2643-2648 (2004).

[11] R. Lu, W. W. Li, B. Mizaikoff, A. Katzir, Y. Raichlin, G. P. Sheng, and H. Q. Yu, Nature Protocols 11, 377-386 (2016).

[12] Y. Raichlin, D. Avisar, L. Gerber, and A. Katzir, Vibr. Spectrosc. 73, 67-72 (2014).

[13] S. MacDonald, K. Michel, D. LeCoq, C. Boussard-Pledel, and B. Bureau, Opt. Mater. 25, 171-178 (2004).

[14] N. Hô, M. C. Phillips, H. Qiao, P. J. Allen, K. Krishnaswami, B. J. Riley, T. L. Myers, and C. Anheier, Jr., Opt. Lett. 31, 1860-1862 (2006).

[15] I. D. Aggarwal and G. Lu, Fluoride Glass Fiber Optics (Academic Press, New York, 1991).

[16] J. Lucas and X. H. Zhang, J. Non-Cryst. Solids 125, 1-16 (1990).

[17] L. Le Neindre, F. Smektala, K. Le Foulgoc, X. H. Zhang, and J. Lucas, J. Non-Cryst. Solids 242, 99-103 (1998).

[18] R. Götz, B. Mizaikoff, and R. Kellner, Appl. Spectrosc. 52, 1248-1252 (1998). 
[19] B. Mizaikoff, R. Götz, and R. Kellner, Proc. SPIE 2508, 253 (1995).

[20] A. Wilk, J. C. Carter, M. Chrisp, A. M. Manuel, P. Mirkarimi, J. B. Alameda, and B. Mizaikoff, Anal. Chem. 85, 11205-11210 (2013).

[21] C. R. Young, N. Menegazzo, A. E. Riley, C. H. Brons, F. P. DiSanzo, J. L. Givens, J. L. Martin, M. M. Disko, and B. Mizaikoff, Anal. Chem. 83, 6141-6147 (2011).

[22] B. Mizaikoff and B. Lendl, in: Handbook of Vibrational Spectroscopy, edited by J. M. Chalmers and P. R. Griffiths (Wiley \& Sons, New York, 2002), Vol. 2, p. 1560.

[23] C. Charlton, M. Giovannini, J. Faist, and B. Mizaikoff, Anal. Chem. 78, 4224-4227 (2006).

[24] X. Wang, S. S. Kim, R. Roßbach, M. Jetter, P. Michler, and B. Mizaikoff, Analyst 137, 2322-2327 (2012).
[25] X. Wang, J. Antoszewski, G. Putrino, W. Lei, L. Faraone, and B. Mizaikoff, Anal. Chem. 85, 10648-10652 (2013).

[26] X. Wang, M. Karlsson, P. Forsberg, M. Sieger, F. Nikolajeff, L. Österlund, and B. Mizaikoff, Anal. Chem. 86, 8136-8141 (2014).

[27] P. Wang, W. Bohr, M. Otto, K. Danzer, and B. Mizaikoff, Anal. Bioanal. Chem. 407, 4015-4021 (2015).

[28] D. T. D. Childs, R. A. Hogg, D. G. Revin, I. U. Rehman, J. W. Cockburn, and S. J. Matcher, Appl. Spectrosc. Rev. 50, 822-839 (2015).

[29] S. Lüdeke, M. Pfeifer, and P. Fischer, J. Am. Chem. Soc. 133, 5704-5707 (2011).

[30] E. J. Castillo, J. L. Koening, J. M. Anderson, and J. Lo, Biomaterials 5, 319-325 (1984).

[31] R. M. Gendreau, Appl. Spectrosc. 36, 47-49 (1982). 